\title{
Celebraciones escolares, ifiestas cívicas? El tratamiento escolar del 9 d'Octubre y del Día de la Constitución en las aulas valencianas de Educación Primaria
}

\section{David Parra Monserrat ${ }^{1^{*}}$ Josep Ramon Segarra Estarelles*}

Departament de Didàctica de les Ciències Experimentals i Socials

Universitat de València

Resumen: El 9 de octubre y el 6 de diciembre se conmemoran dos acontecimientos históricos considerados relevantes para la sociedad valenciana actual: el Dia de la Comunitat Valenciana y el Día de la Constitución. A partir de los distintos modos como se trabajan o celebran en las aulas valencianas de Educación Primaria dos hechos con una carga histórica y cívica tan fuerte, pretendemos investigar la compleja articulación que se da entre ciudadanía, historia e identidades, prestando especial atención al significado de las diferencias y similitudes entre ambos casos.

Palabras clave: Didáctica de las Ciencias Sociales, ciudadanía, educación, conmemoraciones, identidades.

Abstract: On 9 October and 6 December two events considered relevant for the current Valencian society are celebrated: the Day of the Comunitat Valenciana and the Spanish Constitution Day. Studying the treatment of these festivities in Valencian primary schools, we try to investigate the complex articulation among citizenship, history and identities, paying special attention to the significance of the differences and similarities between them.

Key Words: Social Sciences Education, Civic responsibility, Education, Celebrations, Identities.

(Fecha de recepción: junio, 2012, y de aceptación: septiembre, 2012)

DOI: 10.7203/DCES.26.1927

\footnotetext{
$1 *$ El artículo se enmarca en el proyecto de investigación «De la dictadura nacionalista a la democracia de las autonomías: política, cultura, identidades colectivas» (HAR 2011-27392), financiado por el Ministerio de Economía y Competitividad.
} 


\section{Introducción}

Buena parte de las fiestas o conmemoraciones de carácter cívico-político remite a acontecimientos que, en muchas ocasiones, suelen tener algún tipo de valor fundacional para la comunidad concernida. En este sentido, el 9 de octubre y el 6 de diciembre se conmemoran dos hechos históricos considerados, a priori, relevantes para la sociedad valenciana actual: la conquista de Valencia por parte del Rey Jaume I, que ha dado lugar a la festividad o Día de la Comunitat Valenciana, y la celebración del referéndum que, en 1978, condujo a la aprobación de la Constitución vigente.

Pese a su diferente significado cultural e histórico, los dos hechos han sido presentados, con frecuencia, como el nacimiento de un nuevo orden social y político que, con mayor o menor intensidad, ha contribuido a dar forma a concepciones identitarias y ciudadanas.

La sociología, la antropología, la historia y las ciencias de la educación coinciden en que el análisis del impacto y del grado de difusión de este tipo de festividades en el conjunto de la sociedad no puede limitarse al estudio de su dimensión puramente institucional, por lo que resulta imprescindible abordar estos fenómenos a partir de un amplio abanico de ámbitos y, en la medida de lo posible, de zonas geográficas. De este modo, puede resultar de enorme relevancia averiguar el eco que tienen en la prensa, ver si conllevan la realización de actos en el ámbito públi- co (y, si es así, si existe un determinado "ritual cívico"), comprobar quiénes participan en su celebración y de qué modo (desfiles, procesiones, manifestaciones de carácter popular), analizar el tipo de discursos que hay detrás de la fiesta (historicistas, costumbristas, puramente lúdicos), etc. (Corbin, Gérôme y Tartakowsky, 1994; Edensor, 2002).

Dentro de ese complejo estudio de festividades como las que aquí nos ocupan, debemos destacar el papel desempeñado por la escuela, un ámbito que no está al margen de todo este proceso y cuyo análisis ayuda a contextualizar mejor el impacto social de las celebraciones y sus posibles usos públicos tanto dentro como fuera de las aulas.

Históricamente, la celebración de fiestas o conmemoraciones en el ámbito escolar se ha vinculado al proceso de construcción de ciudadanos patriotas. Este tipo de celebraciones debía asegurar la adhesión de los niños al ideal colectivo de la nación y su fidelidad a las élites en el poder. Buena parte de esos actos tendían a la exaltación de las glorias nacionales o a la sensibilización de los futuros ciudadanos para que aprendiesen a honrar los hechos patrióticos considerados importantes para la colectividad $^{1}$. No obstante, a través de los distintos rituales escolares organizados y de las acciones promovidas desde las escuelas en relación con fiestas como el 9 d'Octubre o el 6 de diciembre, se puede desarrollar también una pedagogía ciudadana abierta, crítica e inter-

${ }^{1}$ Véanse al respecto, sobre todo en relación con el 12 de octubre, los trabajos de Carretero, Quiroga, Marcilhacy, del Pozo y Braster citados en la bibliografía. 
cultural, sin ser por ello ajena a valores culturales específicos. Hay que recordar aquí que ninguna comunidad de ciudadanos se ha construido completamente al margen de las formas de vida, de la cultura o de la memoria histórica que sirven para cohesionar una sociedad dada (Romeo, 2004). Lo fundamental, desde nuestro punto de vista, es saber que la escuela no es una mera instancia de reproducción de esos valores sino un ámbito en el que se construyen y legitiman y, por tanto, debería hacerlo de un modo abierto, crítico y no esencialista.

Este artículo, pues, se propone analizar el tratamiento escolar de la educación ciudadana partiendo de los distintos modos como se trabajan o celebran en las aulas valencianas de Educación Primaria dos hechos con una carga histórica y cívica tan fuerte como el Día de la Comunidad Autónoma y el Día de la Constitución. A través de este estudio pretendemos investigar la compleja articulación que se da entre ciudadanía, historia e identidades, prestando especial atención al significado de las diferencias y similitudes entre ambos casos.

Asimismo, el presente texto pretende enmarcarse dentro del debate suscitado en los estudios sobre nación y ciudadanía en relación con la distinción binaria entre nacionalismos cívicos y nacionalismos étnicos, una distinción que ha llevado a muchos autores a distinguir entre comunidades e identidades étnico-culturales, supuestamente basadas en un pasado común y unas raíces muy lejanas, y comunidades e identidades cívico-políticas, fundamentadas teóricamente en la adquisición de la ciudadanía (Brubaker, 2000). Se trata, por tanto, de prestar atención a la complejidad y ambivalencias inscritas en la práctica de la educación en un conjunto de valores, símbolos y memorias compartidas; una serie de elementos sin los cuales no es pensable la ciudadanía en nuestra sociedad pero que, inevitablemente, comportan un sentido del arraigo que la escuela debe proveer y que, con frecuencia, no es ajeno a la vieja función nacionalizadora de la institución escolar.

Nuestra intención, pese a todo lo señalado, no es presentar una crítica paralizante, sino profundizar en las implicaciones culturales e identitarias que, nos guste o no, la educación ciudadana comporta; lo que nos permitirá enfocar con mayor rigor el compromiso cívico de la tarea docente.

\section{Una aproximación a las fiestas escolares: metodología y fuentes uti- lizadas}

Para llevar a cabo este estudio, hemos recurrido, en primer lugar, al análisis de unos cuestionarios repartidos entre el alumnado durante el periodo de prácticas que se desarrolla en el tercer curso del Grado de Maestro en Educación Primaria. Estas encuestas se centraban, fundamentalmente, en el modo como se han llevado a cabo estas celebraciones, el tipo de actos realizados, su vinculación con los contenidos curriculares o sus implicaciones políticas más o menos explícitas. El cuestionario fue contestado, de manera anónima y voluntaria, por 25 alumnos y alumnas que cursaban tercero de 
Magisterio durante el curso académico 2011-2012 y por 16 maestros y maestras en activo que, muy generosamente, les dieron cuenta del tipo de actividades que realizaban, en relación con las celebraciones que nos ocupan, tanto en el aula como en el centro al que pertenecían. Esta muestra es representativa de 45 aulas y de 36 centros ubicados en distintas poblaciones de la provincia de Valencia, especialmente de la capital y su área metropolitana ${ }^{2}$.
Por otra parte, esta investigación se ha fundamentado también en la información que los centros escolares, los docentes y las asociaciones de madres y padres han colgado en páginas web y blogs digitales. Gracias al estudio en profundidad de las webs de 60 colegios de Educación Infantil y Primaria correspondientes a 40 municipios de la provincia de Valencia (FIG. I), hemos podido acceder a comentarios y relatos digitales, revistas escolares, listados

\section{Figura I. Municipios de los que proceden las encuestas y webs escolares con referencias a la celebración del 9 d'Octubre y/o del Día de la Constitución.}
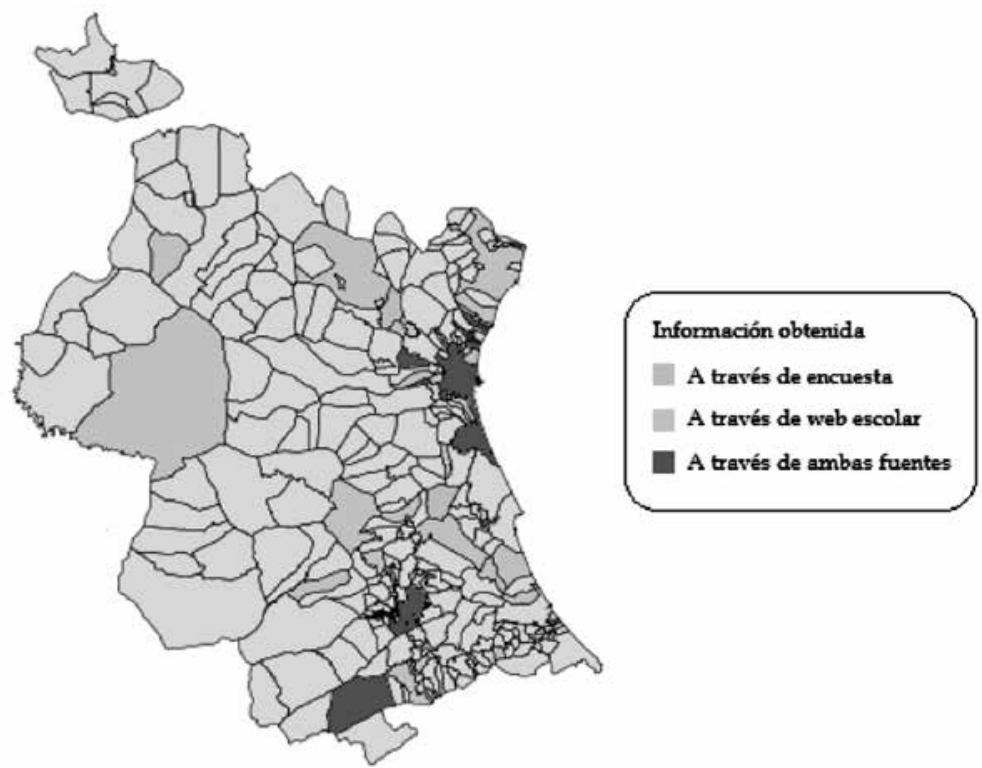

FUENTE: Elaboración propia a partir del estudio de encuestas y páginas webs escolares correspondientes a centros educativos de Alaquàs, Albaida, Albalat dels Sorells, Alboraia, Aldaia, Algemesí, Almàssera, Almussafes, Alzira, Antella, Benagéber, Benetússer, Beniarjó, Benifairó de la Valldigna, Bolbaite, Daimús, El Puig, Faura, L’Alcúdia, L'Eliana, Llíria, Llocnou de Sant Jeroni, Manises, Massamagrell, Meliana, Mislata, Montaverner, Ontinyent, Paiporta, Pobla de Vallbona, Rafelbunyol, Requena, Sagunt, Sant Antoni de Benaixeve, Sedaví, Tavernes Blanques, Tavernes de la Valldigna, Tous, València, Xàtiva, Xeresa y Xirivella.

${ }^{2}$ Debemos señalar que algunos de los alumnos encuestados informaron sobre más de un aula. 
y descripción de actividades, fotografías de espacios y de eventos que no sólo nos han permitido reconstruir con mayor o menor minuciosidad la forma de celebrar una determinada fiesta en la escuela, sino que, además, ponen de manifiesto hasta qué punto la comunidad educativa tiene voluntad de que esas conmemoraciones salgan del espacio escolar y puedan ser vistos por la comunidad virtual.

En relación con esta última cuestión, debemos indicar que el estudio de las páginas web plantea, desde la perspectiva de la investigación, unos obstáculos más que evidentes. El hecho de que en una web aparezcan referencias (textos, fotografías o vídeos) sobre una determinada celebración escolar no significa que dicha celebración se limitase a las actividades allí presentadas. Por otro lado, de la ausencia de información sobre estas festividades en otras 96 webs consultadas no podemos deducir la no celebración de dichas fiestas, por lo que es muy probable que en muchos de los centros en los que, pese a tener página web, no aparecían referencias al 9 d'Octubre y el Día de la Constitución, sí hubiese algún tipo de actividad pese a no quedar reflejado.

A pesar de estas dificultades, consideramos que la muestra de la que disponemos resulta relevante tanto desde una perspectiva cuantitativa como, especialmente, desde una óptica cualitativa, ya que, como veremos a lo largo de estas páginas, buena parte de la información de las distintas webs coincide en resaltar los mismos elementos, lo que nos permite hablar de un elevado grado de saturación de los datos.

\section{El Día de la Constitución en los cen- tros educativos valencianos}

Por lo que respecta al Día de la Constitución, tanto los datos que ofrecen las encuestas como la consulta de los portales web de los colegios muestran la debilidad de la celebración del 6 de diciembre en las aulas valencianas. Del total de 36 centros encuestados, sólo en 12 colegios (menos de la mitad) se llevó a cabo algún tipo de actividad y en ningún caso éstas implicaron una celebración festiva. En los dos casos en los que se realizaron actividades que iban más allá del contexto del aula, éstas consistieron en excursiones a museos y, quizá, en la publicación en las zonas comunes del colegio de murales alusivos a la Carta Magna o a los derechos de los niños. De hecho, en uno de los centros encuestados se dedicó la víspera del Día de la Constitución a realizar una salida lúdica a un parque, por lo que, ni en ese caso, aun habiendo suspendido la actividad ordinaria del aula, se programó nada relativo al 6 de diciembre.

Si tomamos en consideración las doce aulas en las que sí se llevaron a cabo actividades relacionadas con el Día de la Constitución, destaca el hecho de que en la mitad de éstas se realizaron tareas de contenido escolar/curricular (normalmente del área de Conocimiento del Medio), lo que suele limitar las referencias a esta fiesta al tercer ciclo de primaria. Así, en la mayoría de los casos no parece que las actividades de aula se acompañasen de una referencia explícita al carácter conmemorativo del 6 de diciembre sino que, más bien, todo apunta a que se realizaban en fun- 
ción de la secuencia de contenidos de los libros de texto o materiales curriculares usados, al margen del calendario de celebraciones. Por ello, en el tipo de actividades realizadas predominan los contenidos conceptuales; casi nunca falta una "explicación introductoria" de carácter histórico o alguna "lectura" alusiva. Cuando se trata de actividades efectuadas al margen de los contenidos curriculares, las tareas adquieren un sesgo más procedimental, como la representación de una sesión de un congreso o asamblea por parte de los alumnos, la elaboración de murales o, directamente, la realización de actividades extraescolares (excursiones, visitas a muesos etc.).

En los pocos casos en los que las actividades hacían referencia al contenido de la Constitución éstas se ocupaban de aspectos como el derecho a la educación o los derechos de los niños, esta última referencia centrada principalmente en la Declaración de 1959 y no en la Constitución de 1978 propiamente dicha. Asimismo, a pesar del predominio de contenidos conceptuales de carácter histórico, en las actividades realizadas se apelaba más bien poco a la historia política y constitucional española. Sólo en cuatro casos se hacía referencia a otras constituciones anteriores (especialmente la Constitución de Cádiz y la de 1931) y únicamente una maestra introdujo la comparación crítica entre el régimen constitucional actual y la Dictadura militar previa. En el resto de casos, la historia constitucional era presentada más bien como la genealogía lineal de la monarquía parlamentaria y la democracia, una caracterización acrítica del régi- men democrático del presente que quizá esté muy determinada por los libros de texto usados. En cualquier caso, algunos profesores encuestados justificaron ese tratamiento de la Constitución en base a la madurez cognitiva de los alumnos de primaria y a sus dificultades para asimilar conceptos abstractos. A nuestro modo de ver, sin embargo, un enfoque completamente aproblemático de la Constitución o actividades meramente informativas planteadas al margen del currículum evaluable corren el riesgo de vaciar de sentido el carácter cívico de este contenido.

Si nos centramos en el estudio de las páginas web, debemos señalar que de las 156 páginas visitadas, sólo 7 hacían referencia al Día de la Constitución. En todas ellas se solía vincular la conmemoración con el estudio de derechos y deberes, la presencia de iconografía nacional (bandera, escudo, mapa) y, en algunos casos, con referencias a la forma y estructura del Estado (lo que en un colegio llevaba a destacar la idea de la unidad nacional pese a la existencia de las autonomías). Sólo en uno de los 7 casos se hacía una referencia histórica para señalar que ésta es la novena Carta Magna del constitucionalismo español y que la primera fue La Pepa.

Finalmente debemos indicar que dos de las escuelas aprovecharon la conmemoración para desarrollar una celebración festiva con participación de todo el alumnado del centro, lo que se concretó en la lectura pública de algunos artículos del código legal y en la realización de piezas de puzle que, al juntarlas, daban lugar a un mural sobre la Constitución que debía colgarse en el hall del centro. 
Volviendo a las encuestas, debemos señalar que éstas proponían que los alumnos en prácticas y los maestros y maestras entrevistados asociasen toda una serie de conceptos a las actividades que realizaron. A pesar del escaso número de respuestas, consideramos que este test de asociación permite apreciar tendencias significativas que refuerzan lo que ya hemos apuntado (Fig. II).

\section{FigurA II. Respuestas a la pregunta "señala cuáles de estas cuestiones han aparecido en las actividades o actos sobre la Constitución".}

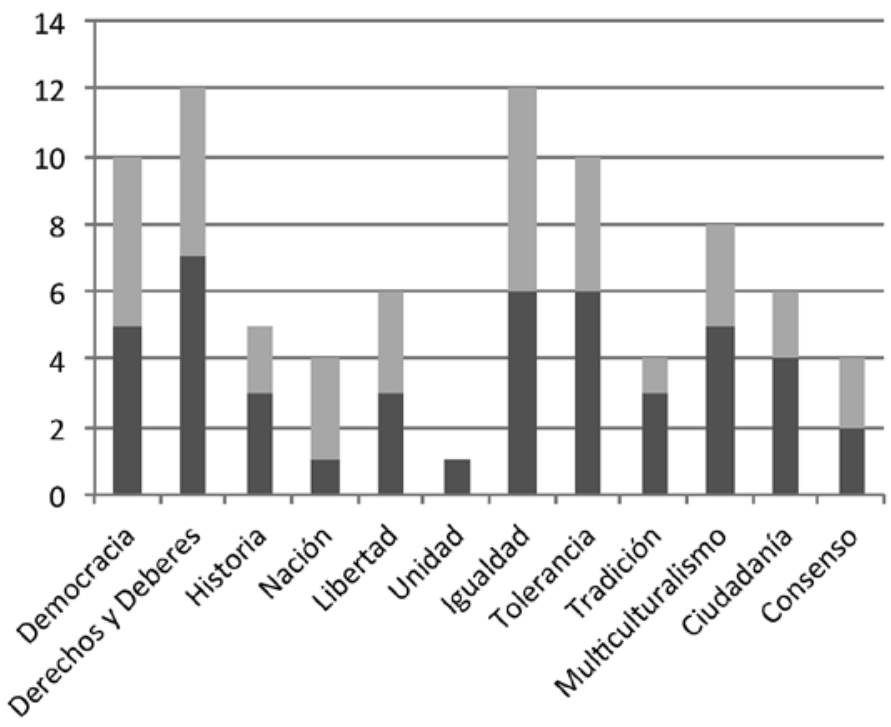

- Respuestas alumnado en prácticas

- Respuestas maestras / maestros

FUENTE: Elaboración propia a partir del estudio de las encuestas.

Como puede observarse, las respuestas ofrecidas por los maestros y maestras encuestados no difieren de modo llamativo de las respuestas indicadas por los alumnos universitarios en periodo de prácticas que actuaron como informantes. Por lo general, se pone el énfasis en conceptos que se corresponden con el campo conceptual de la ciudadanía: "Democracia", "Derechos y Deberes" o "Tolerancia"; asimismo también se observa un cierta tenden- cia a hacer una lectura progresista de la Constitución. En este sentido nos parece significativa la alta valoración del concepto "Igualdad", que duplica el número de respuestas afirmativas del concepto "Libertad". Aunque la "igualdad" podría ser interpretada en dos direcciones diferentes pero no necesariamente contradictorias entre sí (en el sentido de igualdad social o en un sentido cultural de uniformidad). Todo parece indicar que las respuestas tien- 
den a esquivar los componentes más nacionalistas o tradicionales que también se pueden derivar de la Constitución ("Historia", "Nación", "Tradición", "Unidad" e incluso "Consenso"). Muy probablemente, el rechazo a esta lectura de la Carta Magna se encuentra reforzado por el carácter no festivo que, en general, caracteriza al modo en como es abordada la constitución en las aulas valencianas.

No obstante, a nuestro modo de ver, el conjunto de los valores señalados y, en especial, el distanciamiento respecto a las lecturas más nacionalistas del código de 1978, pueden ser interpretados también como muestra de una tendencia a esquivar los temas que han estado más vinculados a polémicas públicas en la lectura de la Constitución. En este sentido, habría que recordar los debates acerca del "patriotismo constitucional" y su énfasis en la unidad y la nación como conceptos fundamentales de la Constitución de 1978 (Balfour y Quiroga, 2007). Así se entiende mejor el sesgo más progresista que preside el enfoque de la Constitución por parte de los maestros y maestras, pero también su tendencia a despolitizar el tratamiento escolar de este tipo de contenidos de carácter cívico. En cierto modo, desde nuestro punto de vista, el debate sobre el "Patriotismo Constitucional" del primer lustro del siglo XXI, en la medida que supuso una lectura "renacionalizadora" de la Constitución, ha podido contribuir a desincentivar su tratamiento escolar en las aulas valencianas, no porque el Día de la Constitución se celebre menos sino porque amortigua el interés de la comunidad educativa por un tema considerado "politizado" y polémico.

\section{El 9 d'Octubre: principales activi- dades y finalidades socioeducativas}

Por contraste con lo que acabamos de ver, de los datos que recogen las encuestas, así como de la consulta de las webs de los colegios, se desprende la importancia en la vida escolar valenciana de la celebración del 9 d'Octubre. Del total de 36 colegios encuestados, un $80 \%$ (29 centros) realizó algún tipo de actividad relativa a esta festividad, mientras que solamente 4 de las escuelas encuestadas no hicieron nada. Entre aquellos colegios que sí celebraron el Día de la Comunidad Autónoma, más de la mitad (16 centros) realizaron algún tipo de celebración global que involucró a todo el colegio, además de actividades de aula relativas al mismo tema. Hay que subrayar que, en un número difícil de determinar (pero, sin duda alguna, mayoritario), la celebración del 9 d'Octubre adquirió un carácter propiamente festivo. Este aspecto se confirma con claridad en los documentos, imágenes y fotografías que muchos centros ofrecen en sus portales web, un conjunto de fuentes que nos ha permitido categorizar el tipo de actividades del siguiente modo (FIG. III).

En primer lugar, tanto las encuestas como las páginas web y/o blogs escolares nos indican que una parte importante de los centros (más del $60 \%$ en el caso de las encuestas y cerca del $40 \%$ en el de las webs) realizaron talleres y actividades de plástica durante la con- 


\section{Figura III. Tipo de actividades presentes en las encuestas y páginas web escolares (en \%)}

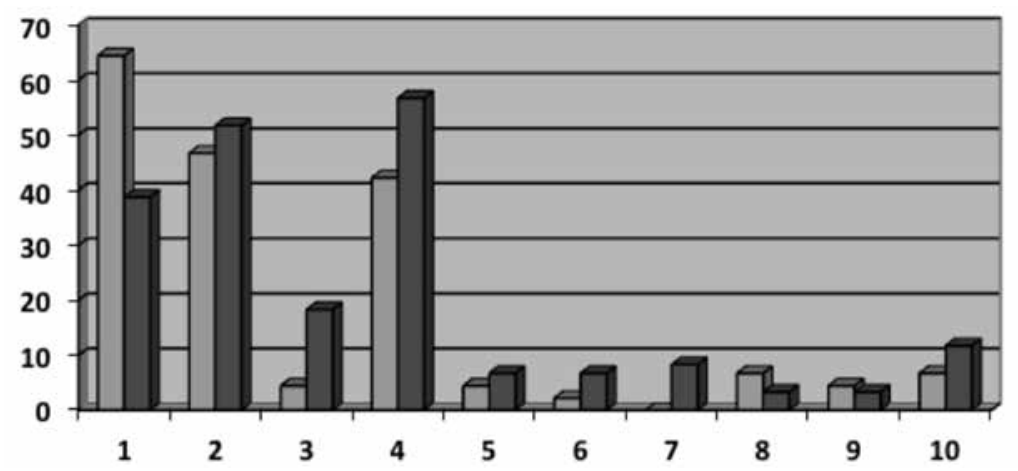

口Encuestas

口Webs

1. Talleres y actividades de plástica (confección de murales, carteles conmemorativos, etc.).

2. Presentación del relato de los principales acontecimientos (históricos o legendarios) relacionados con la festividad.

3. Celebración de la "Mocadorà".

4. Actividades en el patio (desfiles, recreaciones, bailes, etc.).

5. Actuaciones de marionetas sobre Jaume I y la conquista de la ciudad de Valencia.

6. Ferias gastronómicas, chocolatadas.

7. Concursos artísticos / literarios.

8. Salidas extraescolares (paseos históricos, excursiones).

9. Celebración fuera del espacio escolar (conciertos, murales en las calles).

10. Otras (generalmente vinculadas con la lengua desde una perspectiva reivindicativa).

memoración de este evento. Así, fue frecuente la realización de murales y carteles conmemorativos que, posteriormente, eran colgados en las aulas o en los pasillos de la escuela; la confección de banderolas moras y cristianas, de escudos y espadas o de toda una serie de retratos del Rey Jaume I en los que el monarca aparecía rodeado de toda una simbología característica que, en general, tendía a ensalzar su figura.

Igualmente importantes fueron las actividades destinadas a presentar y difundir el relato de los principales acontecimientos (históricos o legen- darios) relacionados con la festividad (algo que encontramos en el $47 \%$ de los cuestionarios y en el $52 \%$ de las webs). Dentro de este tipo de actividades, lo más habitual era la lectura o copia de textos sobre la vida de Jaume I y, en especial, sobre la conquista cristiana de la ciudad de Valencia (muchas veces acompañados de una exaltación de la Señera y del proceso de conquista). En relación con estos ejercicios debemos destacar también la habitual elaboración de aucas sobre la vida del rey (sobre todo en segundo y tercer ciclo) que, generalmente, solían ser colgadas 
en las paredes del aula o del centro para que el resto de la comunidad educativa pudiera leerlas.

El relato mítico o histórico difundido a través de estas actividades solía vincular el 9 d'Octubre con las tradiciones y la identidad valenciana hasta el punto de que, en algunos casos, se llegaba a afirmar que "el objetivo general es el conocimiento del hecho histórico que comportó el nacimiento del actual pueblo valenciano", reduciendo a la mínima expresión o directamente eliminando las posibles influencias de la historia anterior a la llegada de los cristianos.

Como se confirma también en los certámenes literarios que muchos centros organizaban con ocasión de este día, el tema más recurrente era la vida del rey. Generalmente se describía su niñez (que permitía presentarlo como un muchacho listo, osado y valiente que ya tenía dotes de líder), sus hazañas (con numerosas referencias a batallas y conquistas) y toda una serie de datos biográficos que, presentados acríticamente y con un sentido claramente historicista, tenían como finalidad la exaltación de su "gran labor". De este modo, Jaume I era presentado, tanto por los materiales preparados por muchos docentes como por las redacciones elaboradas por algunos alumnos, como "un bon home i un bon rei", cuyo nacimiento fue "un hecho providencial" y cuya muerte provocó que "durant quatre dies a la ciutat sols es van sentir crits i plors". Todo esto, además, reforzado por ilustraciones con una iconografía tendente a reforzar el carácter heroico del monarca (a caballo, con su espada y su yelmo, etc.) o por leyendas que, como la del murciélago que lo previno del ataque moro, le daban a la historia y al personaje un toque providencial más que evidente.

Resulta llamativa la gran ausencia de los "otros", los andalusíes, en todo este relato histórico. Muy pocas encuestas y webs hablan de los moros y, cuando lo hacen, es para señalar que, pese a la resistencia inicial, finalmente fueron expulsados. Ninguna referencia, pues, al drama del exilio o al fenómeno de conversiones forzosas iniciado tras el proceso de conquista.

En tercer lugar, destaca la celebración de la "Mocadorà" y del Día de Sant Donís, una versión tradicional y de ámbito local del "día de los enamorados" que aparece mencionada en el 4,4\% de las encuestas y el $18,3 \%$ de las webs. Generalmente vinculada con la época de la conquista y el 9 d'Octubre, este evento suele dar lugar a un taller de cocina en el que los niños y niñas hacen caramelos o mazapanes con formas de frutas y los envuelven en pañuelos de papel de seda para, teóricamente, regalarlos a la persona amada.

Un $42,2 \%$ de los cuestionarios y un $56,7 \%$ de los blogs y webs indican que las escuelas realizaron numerosas actividades, sobre todo de expresión artística y corporal, en el patio y/o en el resto del centro. Así, es muy habitual que los niños y niñas se disfracen de moros y cristianos y protagonicen desfiladas y marchas, que organicen "danzas medievales" y bailen en grandes grupos o incluso que recreen o escenifiquen el hecho de la conquista, la lucha entre las tropas moras y las cristianas o toda una serie de "tradiciones de nuestra 
Comunidad" relacionadas con el acontecimiento en cuestión.

Entre los bailes mencionados, podemos destacar por su asiduidad la canción titulada "Jaume I tenia cent soldats", "Hi havia tres tambors" (interpretada, habitualmente, con tambores y escenografía castrense) o "Eram sam sam", canciones a las que, en ocasiones, se añadía la célebre "Paquito el Chocolatero".

Aunque de importancia menor, debemos destacar también las actuaciones de marionetas sobre Jaume I y la conquista (presentes en cerca del 5\% de las encuestas y el 7\% de las webs), la realización de ferias gastronómicas y/o chocolatada, la celebración de concursos o certámenes artísticos y literarios, la programación de salidas extraescolares (generalmente visitas o excursiones con finalidad histórico-artística en las que, en ocasiones, se recreaban los acontecimientos del 9 d'Octubre por parte de actores y del propio alumnado) o incluso la celebración fuera del espacio escolar en colaboración con los ayuntamientos u otros organismos públicos o privados. En relación con esta última categoría podemos destacar el caso de dos centros que participaron en un concierto de folk valenciano organizado por el consistorio en el centro cívico y social del municipio o el de varias escuelas que elaboraron grandes murales conmemorativos que fueron expuestos en la vía pública.

Finalmente, un $6,7 \%$ de las encuestas y un $11,7 \%$ de las páginas web introducen otro tipo de actividades generalmente vinculadas a la lengua desde una perspectiva reivindicativa. Así, son varios los casos en los que se realiza un correllengua y se lee un manifiesto por el valenciano, o los que aprovechan el Dia de la Comunitat para estudiar la vida y la obra de escritores e intelectuales como Enric Valor o Manuel Sanchis Guarner.

Gracias al estudio de todas estas actividades hemos podido constatar la existencia de una sinergia entre la celebración del centro y las actividades de aula (incluidas o no en el currículum), ya que cuando existe una celebración a nivel de todo el colegio ésta tiene un efecto de arrastre sobre las tareas escolares de todos los ciclos y etapas.

Puede concluirse, además, que el carácter festivo de la celebración del 9 d'Octubre parece un factor decisivo a la hora de valorar su peso en el calendario escolar. En este sentido se constata un cierto grado de permeabilidad de la escuela respecto a su contexto social más amplio, no porque la celebración del Día de la Comunidad Valenciana tenga un arraigo sociocultural generalizado en todo el territorio, sino porque en la ciudad de Valencia y su entorno metropolitano (de donde mayoritariamente son representativas las encuestas y, en menor medida, las webs) esta celebración sí goza de cierta tradición, vinculada a la fiesta de Sant Donís y a otras tradiciones locales que remiten a símbolos históricos fuertemente arraigados (como la figura del rey Jaume I o la efeméride de la conquista cristiana de la ciudad de Valencia en 1238). En cierto modo, el establecimiento del Día de la Comunidad Autónoma el 9 de octubre ha tendido a usufructuar el valor simbólico que la fecha posee en la ciudad de Valencia y su entorno metro- 
politano, pero no en el conjunto del territorio de la Comunidad Valenciana ${ }^{3}$ (Archilés y Martí, 2004; Archilés, Martí, García y Andreu, 2011).

La existencia de cierta tradición en la celebración del 9 d'Octubre en el entorno sociocultural de los colegios no es el único factor que explica su relevancia en el calendario escolar. A la luz de los datos de nuestra encuesta se puede apreciar que, cuando no existe celebración a nivel de centro, hay una parte de los maestros y maestras (un $24 \%$ según los cuestionarios) que lleva a cabo algún tipo de actividad relacionada con la efeméride en sus aulas y, además, independientemente del ciclo. Esto nos indica que en la comunidad educativa valenciana existe mayoritariamente un compromiso con la conmemoración del 9 d'Octubre que va más allá de la relevancia social o institucional de la celebración. Esta conclusión es coherente con los resultados que ofrecimos en un trabajo anterior, en el que pudimos comprobar que el calendario escolar de celebraciones en los colegios valencianos introduce algunas fiestas validadas socialmente a la vez que presenta una cierta especificidad y una clara orientación progresista o modernizante (Parra y Segarra, 2011, p. 77). En este sentido, consideramos que la importancia de la celebración escolar del 9 d'Octubre puede tener uno de sus impulsos en el compromiso "autonomista" y progresista, hegemónico en la comunidad educativa valenciana desde la Transición.

A pesar de todo lo señalado, el compromiso cívico que parece apuntar la celebración escolar del 9 d'Octubre no se traduce en actos que vayan más allá del espacio estrictamente escolar. Sólo en 4 casos (en los que se realizaron actos en la calle con la voluntad de llegar a una audiencia mayor) la celebración adquirió un sentido verdaderamente público, algo que, a menudo, parece depender más de la iniciativa de determinados ayuntamientos que de propuestas nacidas en el seno de la comunidad educativa. Esto, además, contrasta llamativamente con la dimensión eminentemente pública de otras fiestas muy relevantes en el calendario escolar como las Fallas o el Carnaval, que implican habitualmente desfiles y celebraciones por las calles de la localidad (Parra y Segarra, 2011).

Si volvemos al interior de la escuela, podemos afirmar también que, pese a lo señalado en relación con el compromiso de los docentes, todo parece apuntar a un déficit de contenido cívico en la celebración del Dia de la Comunitat, ya que las actividades analizadas confirman que se tiende a una banalización perfectamente ejemplificada con los talleres de plástica o con los desfiles de moros y cristianos (citados en 22 de las 60 páginas web en las que encontramos referencias al 9 d'Octubre).

Esto, vinculado con el tipo de relato histórico mayoritariamente difundido,

${ }^{3}$ En este sentido consideramos que sería muy conveniente llevar a cabo estudios similares en Castellón y Alicante para establecer comparaciones y comprobar si, como suponemos, la celebración del 9 d'Octubre experimenta cambios sustanciales en relación con lo que hemos visto para el caso de la provincia de Valencia. 
nos lleva a concluir que en muy pocas ocasiones se ha pensado esta fiesta en términos de educación intercultural, ya que no sólo se ignoran las potencialidades de esta conmemoración para tratar la diversidad, sino que, además, el tratamiento que en algunos casos se hace de los "otros", sin ser negativo, puede acabar reforzando las ideas de alteridad sobre lo arabo-musulmán más o menos arraigadas en el alumnado (Delval, 2006; Valls, 2008).

Queremos destacar por ello que, pese a ser una minoría, hay algunas escuelas que sí introducen un enfoque intercultural en la celebración del 9 d'Octubre. Así, hay casos en los que se recrea la llegada de Jaume I a tierras valencianas y se destaca el pacto de amistad y convivencia entre moros y cristianos, remarcándose el papel de la lengua como foco de entendimiento. En otros casos, se explica el origen de la fiesta, se compara con otras fiestas similares de otras comunidades autónomas y se trabaja la "herencia que nos han dejado la cultura cristiana y la árabe para comprender lo que somos hoy" (tanto desde una perspectiva lingüística, como artística, gastronómica o de costumbres). Es habitual en estos casos que la festividad se vincule con conceptos como "pluralidad", "convivencia", "respeto", "sentimiento" o "empatía", lo que confirma que es posible hacer una fiesta arraigada en las tradiciones y con un marcado carácter cívico.

\section{Conclusiones}

Los datos analizados a lo largo de estas páginas ponen de manifiesto, en primer lugar, diferencias claras entre la débil celebración del Día de la Constitución y la mayoritaria celebración del 9 d'Octubre. Por lo que se puede comprobar en los datos de este estudio, la no existencia de una celebración social de carácter folklórico o cultural asociada al 6 de diciembre parece un hándicap insuperable: sin ese elemento externo que haga de arrastre, la escuela no acaba de hacerse permeable. Lo que parece decisivo es que el 9 d'Octubre está en el calendario escolar y el 6 de diciembre no, lo que tiene relación con una cierta ósmosis con respecto al contexto sociocultural (Valencia y su área metropolitana) y a la orientación "autonomista" (y en muchos casos progresista) que desde la Transición ha caracterizado a muchos docentes valencianos. Las diferencias marcadas en la celebración de ambas fiestas no habría que interpretarlas en términos de debilidad del "sentimiento constitucional" o de debilidad de la "identidad nacional española" en la Comunidad Valenciana, puesto que lo que parece determinante en la celebración del 9 d'Octubre es, por un lado, el "autonomismo" que se enmarca en los valores de la Constitución de 1978 y, por otro, el peso de la identidad regional valenciana que se entiende dentro del marco de la nación española (Archilés y Martí, 2004).

En segundo lugar, hemos observado una tendencia a huir de las implicaciones ciudadanas y políticas de estas dos celebraciones; es decir, una tendencia a despolitizar este tipo de festividades escolares. Así, cuando estas celebraciones entran en el mundo escolar, se produce un filtraje que elimina los componentes políticos que pueden sus- 
citar polémicas y que, desgraciadamente, conduce a una educación ciudadana muy pobre.

En este sentido, nos hemos encontrado con centros en los que la no celebración de festividades como el 9 d'Octubre se amparaba en un ideario implícita o explícitamente apolítico. En el caso concreto de una encuesta, el alumno informante señalaba que el colegio, laico y democrático, huía de cuestiones políticas para evitar roces o conflictos. Pese a ser un caso particular, consideramos que apunta a una tendencia creciente según la cual la escuela debe ser "neutra" para ser respetuosa. Esto explica que buena parte de las celebraciones tiendan, como hemos visto, a banalizar los actos introduciendo actividades de carácter lúdico y festivo (bailes, desfiles, decoración del centro) que huyen de las implicaciones ciudadanas de fiestas como el 9 d'Octubre. Esto, además, se ve reforzado por el hecho de que el arraigo tradicional de esta fiesta en el entorno metropolitano de la ciudad de Valencia y, por tanto, en el contexto sociocultural de los centros analizados, propicia que el contenido cívico de la celebración se transmute en un contenido de "fiestas y tradiciones", con toda la tendencia banalizadora e irreflexiva con la que suelen trabajarse esos contenidos (Parra y Segarra, 2011).

Consideramos, pues, que el tratamiento escolar del 9 d'Octubre y del Día de la Constitución en los centros valencianos de Primaria plantea escasas conexiones con una educación ciudadana. Aunque no por ello queremos dejar de señalar que los pocos centros que sí lo intentan demuestran que, cuando se da la conexión entre lo cívico y lo cultural-tradicional, cuando se trabaja de manera reflexiva y crítica, se puede dar fuerza y relevancia a enfoques interculturales muy ricos y útiles para la construcción de la ciudadanía.

\section{ANEXO. Modelo de encuesta realizada a alumnado en prácticas y docentes en activo}

\section{ENQUESTA SOBRE FESTES I CELEBRACIONS}

1. A l'escola on estàs fent les pràctiques s'ha commemorat d'alguna manera el Dia de la Constitució (6 de desembre)? (Marca amb negreta una o diverses opcions)

a) No, no s'ha fet res.

b) Sí, s'han fet activitats a l'aula sobre el tema.

c) Sí, s'ha organitzat alguna activitat a l'escola sobre el tema.

2. S'ha treballat com a contingut escolar o s'ha celebrat al marge de les activitats diàries de classe? 
3. Quin tipus d'activitats s'han realitzat? Descriu-les breument

4. Com ja saps, al llarg de la història contemporània hi ha hagut diverses constitucions a Espanya. S'ha fet referència en algun moment a alguna d'elles? De forma crítica o com a referent?

5. S'ha fet referència a articles concrets de la constitució actual? Recordes de què parlaven?

6. Assenyala amb negreta quines d'aquestes qüestions han aparegut durant les activitats o actes sobre la constitució:

$\square$ Democràcia
$\square$ Drets i deures
$\square$ Història
$\square$ Nació

$\square$ Llibertat
$\square$ Unitat
$\square$ Igualtat
$\square$ Tolerància

$\square$ Tradició
$\square$ Multiculturalisme
$\square$ Ciutadania
$\square$ Consens

7. Pots esbrinar si a l'escola on estàs fent les pràctiques s'ha commemorat d'alguna manera el 9 d'octubre)? (Marca amb negreta una o diverses opcions)

a) No s'ha fet res

b) S'han fet activitats a l'aula sobre el tema

c) S'ha organitzat alguna activitat a l'escola sobre el tema

8. S'ha treballat com a contingut escolar o s'ha celebrat al marge de les activitats diàries de classe?

9. Quin tipus d'activitats s'han realitzat? Descriu-les breument

10. S'ha parlat de l'Estatut d'Autonomia durant els actes o les activitats?

\section{Referencias bibliográficas}

ARCHILÉS, F. y MARTÍ, M. (2004).

La construcció de la regió com a mecanisme nacionalitzador i la tesi de la dèbil nacionalització espanyola. Afers. Fulls de recerca $i$ pensament, 48, 265-308.

ARCHILÉS, F., MARTÍ, M., GARCÍA CARRIÓN, M. y ANDREU
MIRALLES, X. (2011). Ser de Castelló. La identitat local en l'època contemporània (c. 1880-1936). Castelló de la Plana: Fundación Dávalos-Fletcher.

BALFOUR, S. y QUIROGA, A. (2007). ESPAÑA REINVENTADA. NACIÓN E IDENTIDAD DESDE LA TRANSICIÓN, Barcelona: Península. 
BRUBAKER, R. (2000). Mitos y equívocos en el estudio del nacionalismo. En Hall, J. (ed.), Estado y nación. Ernest Gellner y la teoría del nacionalismo. Madrid: Cambridge University Press, 352-395.

CARRETERO, M. (2007). Historia y patria en el calendario. En Documentos de identidad. La construcción de la memoria histórica en un mundo global. Buenos Aires: Paidós, 199-271.

CORBIN, A., GÉRÔME, N. y TARTAKOWSKY, D. (dirs.) (1994). Les usages politiques des fêtes aux $X I X^{e}-X X^{e}$ siècles. París: Sorbonne.

DELVAL, J. (2006). Hacia una escuela ciudadana. Madrid: Morata

EDENSOR, T. (2002). National identity, popular culture and everyday life. Oxford: Berg.

MARCILHACY, D. (2010). La fiesta de la raza como ideal nacional institucionalizado. En Raza hispana. Hispanoamericanismo e imaginario nacional en la España de la Restauración. Madrid: Centro de Estudios Políticos y Constitucionales, 325-583

PARRA MONSERRAT, D. y SEGARRA ESTARELLES, J. R. (2011). Cultura y pertenencia: el tratamiento didác- tico de contenidos histórico-culturales en las aulas valencianas de Educación Infantil y Primaria. Didáctica de las Ciencias Experimentales y Sociales, 25, 65-83.

POZO ANDRÉS, M.M. y BRASTER, J. (1999). The Rebirth of the «Spanish Race»: The State, Nationalism, and Education in Spain, 18751931. European History Quaterly, 29 (1), 75-107. http://dx.doi. org/10.1177/026569149902900103

QUIROGA FERNÁNDEZ DE SOTO, A. (2007). Maestros, espías y lentejas. Educación y nacionalización de masas durante la dictadura de Primo de Rivera. En Moreno Luzón, J. (coord.). Construir España: nacionalismo español y procesos de nacionalización. Madrid: Centro de Estudios Políticos y Constitucionales, 183-206.

ROMEO MATEO, M. C. (2004). Discursos de nació i discursos de ciutadania. Afers. Fulls de recerca $i$ pensament, 48, 309-326.

VALLS MONTÉS, R. (2008). La imagen del islam en los actuales manuales escolares españoles de historia. En Cajani, L. (dir.). Conociendo al otro: el Islam y Europa en sus manuales de historia. Santillana, 73-122. 